Kom, 2016, vol. V (2) : 1-21

UDC: 14 Мула Садра Ширази

111 Мула Садра Ширази

28-1 Мула Садра Ширази

DOI: $10.5937 / \mathrm{kom} 1602001 \mathrm{~A}$

Original scientific paper

\title{
An Analysis of the Proofs for the Principality of the Creation of Existence in the Transcendent Philosophy OF Mulla Sadra
}

\author{
Shiraz Husain Agha \\ Faculty of Philosophy, Al-Mustafa International University, \\ Qom, I. R. Iran
}

The Transcendent Philosophy of Mulla Sadra was a complete philosophical system that sought to harmonize various intellectual trends that existed in the Islamic world prior to its genesis. It not only brought new philosophical discussions to the forefront but it also helped solve many of the philosophical problems that had until then not been properly resolved. One of the topics that this school of thought discussed in detail was the subject of creation. This discussion revolves around the question as to what is it that a cause essentially brings into reality? What does a cause do? In this regards there are three views. The first is the view that a cause essentially brings quiddity into reality. The next is that a cause gives existence to quiddity. Mulla Sadra was of the opinion that a cause essentially creates existence and through it, it brings quiddity into reality. In order to substantiate this idea, Mulla Sadra tried to refute the other two possible alternative options. This article seeks to analyze the proofs that he put forward to affirm the principality of the creation of existence. The majority of the proofs have been taken from Mulla Sadra's independent work on this subject, Regarding the Principality of the Creation of Existence. Many of these proofs are problematic and are not compatible with other philosophical principles. Others are sound and can be relied on to substantiate this claim. All in all, it can be said that the principality of the creation of existence is one of the necessary corollaries of the principality of existence itself.

Keywords: Existence, principality, creation, quiddity, reality

Corresponding author: ahmadspapa@gmail.com 


\section{Introduction}

The Transcendent Philosophy of Mulla Sadra was an innovation in Islamic philosophy that brought new philosophical discussions to the forefront, solved many philosophical dilemmas that had not previously been resolved and presented innovative philosophical principles that were interconnected in a systematic whole. The cornerstone of this philosophical system was the principality of existence (Ubudiyyat 2013: 1). This idea and others such as the gradation of existence, the copulative nature of the existence of the effect, substantive motion, the unity of the known, knowledge and the knower, and the immateriality of the faculty of imagination helped shape a new intellectual outlook that not only directly influenced philosophy but also was significant in other areas of religious study. Thus, the magnitude of this philosophical school of thought cannot be over emphasized - even though Mulla Sadra has been criticized for stealing some of his ideas from others (Fazlur Rahman 1975: 7).

One of the discussions that was of principle importance for Mulla Sadra was the topic of cause and effect. Mulla Sadra discusses this subject in detail in many of his works. Of primary importance for him was the nature of the effect and its relation with the cause (Misbah 1999: 503). The idea that this relation is essential to the existence of the effect and that because of this the existence of the effect is copulative in nature is something that Mulla Sadra describes as "the point where philosophy attains perfection" (Mulla Sadra 1963: II/292). Aside from this, Mulla Sadra also presented many novel solutions to ancient philosophical discussions about causation. One of these discussions was the discussion surrounding "creation" ( $\mathrm{ja} l \mathrm{l})$. When a cause brings an effect into reality what does it essentially do? What does it essentially create? Before the cause brought the effect into reality neither was the existence of the effect real nor was the quiddity of the effect real. Neither of them possessed reality. Neither of them were real entities in the external world. When the cause creates the effect both of them are brought into reality. The question therefore arises as to what has the cause done? Has it essentially brought the existence of the effect into reality, i.e. has it essentially created it and accidentally created the quiddity of the effect and brought it into reality through its existence? Or, has it actually and essentially created quiddity and only accidentally, or metaphorically, created existence? There is also a third option. That is that the cause has given existence to quiddity (Tabatabai 2010:22). This is the problem of " $j a$ 'l" or creation and three of the basic views regarding the subject.

For the sake of brevity, we cannot enter this discussion in detail. There are many discussions here that need to be addressed before we can properly 
understand all of the dimensions of the problem at hand. Nevertheless, this article will attempt to briefly analyze some of the proofs that Mulla Sadra presented to substantiate this idea. The majority of these proofs have been taken from Mulla Sadra's independent work on this subject [Regarding] the Principality of the Creation of Existence (Asalah Ja'l al Wujud). Some of them can also be found in his magnum opus The Four Intellectual Journeys (Asfar al Arbaiah al Aqliyyah).

\section{The Proofs for the Principality of the Creation of Existence}

By way of introduction, something deserves to be mentioned regarding these proofs. First of all, the majority of them work indirectly. Meaning, the majority, if not all of them seek to prove why quiddity is not what has essentially been created. That, coupled with the rejection of the idea that the cause gives existence to quiddity, automatically specifies existence as that which is essentially created. Thus, the proofs work via negativa. In the forthcoming, we will first present a summary of each proof and then we will separately state each one of the explicit and sometimes implicit premises that are necessary in order to properly understand the argument. In order to felicitate understanding, we have also entitled each proof with a title that indicates the axis around which the proof revolves. Also, we will also state any objection that we feel the proof is afflicted with.

\section{The First Proof: Quiddity is Known, in and of itself, without the Help of Anything Else}

If quiddity is what is essentially and principally created, then it would entail that we could not comprehend quiddity without comprehending its cause. However, this is not true. Thus, quiddity is not what is essentially and principally created (Mulla Sadra 2006: IV/182).

1. If quiddity is what is essentially and principally created and existence is created through it, then it would entail that quiddity, in and of itself, has a cause.

This premise is clear. It rests upon the idea that cause and effect are necessary corollaries of one another. If quiddity is the essential effect of something, it naturally follows that the latter is its cause.

2. If quiddty, in and of itself, has a cause, then it would mean that it is impossible to understand quiddity, in and of itself, without that cause. 
The reasoning behind this premise is a famous dictum of Islamic logic which is that things that have causes cannot be understood without the understanding of those causes. The reason for this principle has been stated in numerous ways one of which is the following: if the cause of something that has a cause is not known, then it would lead us to not being sure about the existence of the effect. If we are not sure whether the cause of some effect exits how can we really be sure if the effect exists? It is clear that if we are unsure about the existence of the cause of some being this uncertainty will lead to us being unsure about the existence of the effect of that cause. Thus, in the case where something has a cause we can only be sure about it when we are sure about its cause. In the case at hand, we are assuming that quiddity, in and of itself, has a cause. Thus, based upon the abovementioned principle, we cannot conceive of the quiddity without being sure about its cause. This is how the necessity between the precedent and the antecedent in the minor premise of the abovementioned conjunctive conditional syllogism is proven.

3. However, quiddity, in and of itself, can be understood without the need to understand its cause.

The soundness of this premise can be affirmed simply by referring to the mind and the manner in which it understands quididty, in and of itself. Thus, the basis of this premise is knowledge by presence. This implies that this premise is self-evident and does not need any proof in order to be affirmed (Fayyadhi 2007: 98). We are able to understand quiddity in and of itself without the need to understand anything else whatsoever other than the quiddity, its necessary components and its necessary external attributes. There is a well-known philosophical axiom in Islamic philosophy that states: "Quiddity, in and of itself, is nothing but itself. It neither exists nor does it not exist." This dictum implies that all types of contradictory concepts can be negated from quiddity except those that form its necessary properties. If this dictum is true, then it means that we should be able to negate being an effect from the quiddity taken in and of itself.

\section{Objection}

It is possible that someone may object here by saying: if what you say is true, i.e. existence is what is essentially created and brought into existence through the cause, then based upon this proof, we should not be able to understand existence unless we first understand its cause. However, this is not the case. We can clearly understand existence without taking anything else into consideration. Thus, we have to call into question the necessity between something's having a cause and its not being capable of being understood without that cause. 
Answer

Mulla Sadra answers this question by rejecting the major premise of this objection. Meaning, he says that it is not true that we understand existence without understanding its cause. In fact, Mulla Sadra is of the opinion that existence is not understood at all. This requires an explanation. In the discussion on mental existence in Islamic philosophy there is a long and drawn-out discussion regarding the epistemological value of our acquired knowledge. When we understand something through mental forms - and not directly through intuitive, presential knowledge - what is it that we really understand? Mulla Sadra and the majority of Islamic philosophers are of the opinion that it is quiddity that we understand. Meaning, quiddity enters the mind exactly as it is in the external world. However, existence is not the same. Existence is not understood by the mind exactly as it is in the external world. Why? This is because "being in the external world" is an essential property of existence - based upon the principality of existence. If existence entered the mind exactly as it is in the external world it would have to bring along with itself this property of "being external". This would lead to the mind and reality uniting and fusing together. However, this is impossible as the mind is a depiction of reality, not reality itself. Thus, existence is not understood by the mind through acquired knowledge and if it is to be understood it can only be understood directly through presential knowledge (Tabatabai 2006: 102).

In this way Mulla Sadra is able to answer this objection by calling into question the very idea that existence is understood at all. It is not understood separately from its cause since it is not understood at all.

\section{Critique}

1. One of the principle premises of this argument is the idea that if something has a cause, it is necessary to understand it through its cause and that if the cause is not known, then the effect will also be unknown. From this it follows that if quiddity, in and of itself, was created, it could not be known except through its cause. When we negate the fact that quiddity must be known along with its cause we come to the conclusion that quiddty is not created in and of itself. However, it is possible for us to make the following objections to this principle:

1. First of all, this principle is not self-evident nor has any adequate proof been presented to substantiate it. To the contrary, there are many things that are effects, the existence of which we are sure about without being in any way sure about their causes. For example, we are sure about our own existence 
through direct intuitive knowledge without even being aware about what our causes are, let alone being sure about their existence. It seems that the philosophers who have stated this principle have confused what takes place in the external world and what goes on in the mind. Meaning, they have confused a metaphysical discussion with an epistemological one. It is true that in the external world the effect can only come into existence through its cause. It does not necessarily follow from this that in the mind the same process occurs, as is abundantly clear from the example we have given regarding our own existence.

2. Leaving aside the correctness of this principle, even if we assume that it is correct, it would not be sufficient to prove the idea that quiddity is not created in and of itself. Why? The reason for this is that this principle states that if something has a cause, its existence can only be known through its cause. It does not state that it can only be known in every manner whatsoever through its cause. Thus, it does not want to say that if we want to know what an effect is, then we must be sure of what its cause is or that its cause exists. If this is the content of this principle, then the actual meaning of the abovementioned syllogism would be the following: if quiddity is what is essentially created, then it would be impossible to be sure about the existence of quiddity without being aware that its cause exists. However, we do become sure about the existence of a quiddity without being sure about the existence of its cause. Thus, quiddity is not what is essentially created. Restated in this way the fallacy in the argument becomes abundantly clear. We, in no way, become sure about the existence of a quiddity without having knowledge about anything else. If quiddity is understood, in and of itself, then this is an understanding that is related to what it is not its existence. In other words, this is a simple conceptual knowledge. There is no judgment contained within it. Thus, the major premise of the argument does not negate the antecedent of the minor premise. Consequently, it is irrelevant to the argument.

3. Aside from the abovementioned objections, there is another fundamental objection at play here. That objection is the following: we cannot accept the idea that existence is something that cannot be known through acquired knowledge and that the only way to accept existence is through presential, intuitive knowledge. Why? Because when we refer to the discussion on mental existence, the following points come to mind:

1. First of all, when these proponents of mental existence say that quiddity comes into the mind exactly as it is in the external world they want to say that the concept of quiddity in the mind parallels what it is in the external 
world. This idea should be understood by contrasting it to what it wants to negate. The ideas that this view stands opposed to seek to affirm the fact that quiddity loses something when it enters the mind as a concept or that it does not come into the mind as a concept at all. Thus, the idea of mental existence simply wants to state that, as a concept in the mind, quiddity possesses all of the components that it did when it was a reality in the external world, albeit as concepts, not realities. What is more, when we look at the answers to the objections to mental existence, we see that they reaffirm the idea that quiddity simply exists in the mind as a concept, not as a reality. If this is true, then there is no problem in existence's coming into the mind since it does not lead to the mind and reality actually being united. Rather, it is simply the unification of the reality of the mind and the idea of reality. Hence, we cannot accept the answer of Mulla Sadra to the question put forward against this first proof (Fayyadhi 2008: 94).

Aside from all of these, one can equally object to the answer of Mulla Sadra in the following manner: if existence is really what is essentially created and it can be known through presential knowledge, then we can only understand it through this type of knowledge if we first have presential knowledge of its cause. It is ironic that Mulla Sadra was actually aware of this objection and he sought to answer it by saying that it is not incorrect to say that we are aware of existence without the knowledge of its cause. Rather, whenever if we become intuitively aware of the existence of a possible being, it is through the prior, intuitive awareness of its cause. This is something that seems to go against what we intuitively feel. What is more, explaining this by saying that this knowledge is only subjective in nature only seems to reaffirm the objection itself.

\section{The Second Proof: Quiddity Has Multiple Individuals}

If quiddity is what is essentially created, then it would entail that there would not be multiple individual members of any quiddity. However, there are multiple individual members of some quiddities. Thus, quiddity is not what is essentially created (Mulla Sadra 2006: 183).

1. If quiddity is what is essentially created by the cause, then it would mean something simple in nature is what has been essentially created by the cause. By 'simple' we mean something that has not been accompanied by anything else other than what it is. This premise seems self-evident since under the assumption quiddities, in and of themselves, have been created. Thus, quiddities have been created without being accompanied by anything else. 
2. If something is not accompanied by anything extraneous to its essence then it will not be numerous. The reason for this is the famous philosophical dictum that states: "The simple thing cannot be multiplied or doubled," or that "there is no differentiation in something that is simple, i.e. something that is not accompanied by anything outside its essence". This principle rests upon the idea that a multiplicity of individuals of a certain concept can only come about when that meaning or concept is added to different and varying qualities that bring numerous individuals into existence. For example, Zayd and Amr are two individual human beings. What is it that makes them two human beings? It is not their quality of being human beings; rather, it is the specific attributes that have been added to the universal quality. This is clear since a multiplicity will only arise when there are different units all of which have points of difference from one another.

3. However, it is clear that multiple individuals of certain quiddities have been created. This is because we see that there are a few individuals of certain species the existence of which we have intuitive knowledge about. For example, all of us have an intuitive awareness of the qualities of our soul. One of these qualities is knowledge. Acquired knowledge is a universal concept that has numerous instances and individuals that we are intuitively aware of since we see some of them within us.

Conclusion: Quiddity is not what has essentially been created.

\section{Objection}

Here it is possible that someone may make an objection to the abovementioned proof: what is wrong if we say that although quiddity is what has essentially been created it nevertheless comes to acquire numerous individuals through the numerous relations that it comes to acquire with its cause. Put in this way, this objection seems to want to call into question the idea that if quiddity is what is essentially created, then it would lead to it being a simple being, i.e. the first premise.

Answer

Mulla Sadra was himself aware of this objection and took it upon himself to answer it. The way he answers it is in the following manner: A relation can only lead to the multiplicity of a quiddity if it is multiple. Its multiplicity rests upon the multiplicity of the two sides of the relation. In the case at hand, neither of the two sides can lead to the multiplicity of the relation since the quiddity is still one. Its leading to the multiplicity of the relation will involve 
a viscous circle of causes. The other side of the relation is the cause which is also one thing. Thus, there is no way that the relation can be multiple. Since it is not multiple, it cannot lead to the multiplicity of the quiddity.

\section{Objection}

Another objection can be leveled here. It is possible for someone to say that it is the multiplicity of conditions and materials in which these quiddities come into being that causes them to become multiple. For example, if a man comes into being in America or to certain parents he will be an individual that is different from the human being that came into being in England and to another set of parents. Thus, the conditions surrounding the quiddity and the matter in which it inheres cause it to become distinct from another instance of the same quiddity.

\section{Answer}

Mulla Sadra answers this objection in the following manner:

1. First of all, these conditions and these materials are things that lie outside of the quididty. They are not qualities of this quiddity. In order for them to take the quiddity out of its state of unity and into the state of multiplicity and individuation they have to be qualities of the quiddity that are predictable for it. Since this is not the case there is no way they can make the quiddity multiple.

2. Secondly, even assuming that they are truly qualities of the quiddity they still do not have the ability to take the quiddity out of its state of unity and universality into the state of multiplicity and individuation. The reason for this is that it is only existence that has the quality of giving quiddity individuation. Things other than existence can make a quiddity numerous and multiple but they cannot give it a state of individuation. The reason for this is that everything other than existence is universal in nature, capable of being predicated of more than one thing. If this is so, then it is clear that they cannot lead to something else that is also universal like themselves to become particular.

\section{Critique}

1. If we can prove that the idea that quiddity is what is essentially created leads to it being a simple thing, then it seems that the rest of the abovementioned proof is sound. The crux of the problem is establishing this necessary 
connection. The principle that a simple reality is not capable of having numerous individuals is something that is at least almost self-evident. The crux of the matter is how to prove the former claim. It seems that there is a problem here. To explain, the claim of Mulla Sadra is that existence is created essentially and quiddity is created through it. Meaning, quiddity is created accidentally. In order to prove this claim Mulla Sadra must prove that every other alternative explanation for creation is wrong. One of other alternative options to this claim is that, opposite to his claim, quiddity is what is created essentially and existence is created through it. If the essential creation of quiddity is stated in this manner, then it seems that there will no longer be any necessary connection between the precedent and the antecedent of the minor premise of the argument. The reason for this is that in this case quiddity will no longer be a simple reality that is not accompanied by anything else. Rather, it will be accompanied by existence, i.e. the very thing that, according to Mulla Sadra, is the cause of all individuation and multiplicity.

Although we feel that this objection is true, we would like to credit Mulla Sadra for overlooking it. The reason for this is that although this alternative option exists and it is the responsibility of Mulla Sadra to negate it, none of the adherents of the principality of quiddity say that quiddity is essentially created and existence is created through it. Rather, they say that quiddity is created and existence is not created at all. Neither is it created essentially nor is it created accidentally, i.e. through existence.

2. Another objection that might be leveled here is the following: it seems that two proofs have been combined in this proof. These two should be separated from one another and analyzed independently. The distinction between them lies in the manner in which we state the antecedent of the minor premise of the argument. This minor premise can be formulated in one of the two following manners:

a) If quiddity is what is essentially created, then there would not be any individuals for that quiddity.

b) If quiddity is what is essentially created, then there would be no multiplicity for that quiddity. If we take the second formulation of the premise into consideration, we can make the following objection: while it is true that existence leads to the multiplicity of quiddity and that if existence was not created, the multiplicity that it brings about would not come about for quiddity, nevertheless, quiddity can also come to possess a multiplicity from the addition of other quiddities to it. We agree with the idea that it is only existence that can lead to individuation but even Mulla Sadra cannot argue with the fact that the addition of different quiddities to one quiddity can lead to the multiplicity of the latter. In this case the necessary connection 
between the precedent and the antecedent of the minor premise will be lost. This is because when we say that quiddity is what has been created "in and of itself" we do not imply by this that it is all alone and that no other quiddities accompany it. The phrase "in and of itself" simply means that it is what has been essentially created and other things, i.e. existence, have not been created at all or have been created through it. Hence, it does not state anything regarding other quddities. Thus, the idea of being created essentially does not negate the possibility of being accompanied by other quiddities that might multiply it.

\section{The Third Proof: The Quiddity of the First Effect of God is a Specific Individual}

If quiddity is what has been principally and essentially created, then it would lead to the random choosing of one of many possible things without a valid reason. However, the latter is impossible. Thus, quiddity is not what has been principally created (Mulla Sadra 2006: 184).

1. If quiddity is what has been principally created, then this would be true in all cases. Thus, it would be the quiddity of the first effect of God that was created by Him. However, this first effect of God is one individual being. The quiddity of the first effect is equally related to all of the individuals that are its instances. There is no reason to say that this particular individual deserves to be created more than others. In this case choosing to create one individual to the detriment of the others leads to the choosing of one of many possible options without a valid reason. In other words, quiddity is something universal. It is present in all of its individual members. From this point of view there is no difference between them. Now, when God creates this quiddity $\mathrm{He}$ can choose to create it any of its individual members. The question arises as to why $\mathrm{He}$ creates it in one individual to the detriment of the other. If we say that quiddity is what was essentially created by God there is no reason to say that one individual deserves to be created more than others. Thus, in the case of the first effect of the First Cause, which philosophers admit is one particular individual, this theory will lead to the choosing of one of many possible options without a valid real reason. To explain further, according to the principality of the creation of quiddity, it is quiddity that is created first and existence follows it in creation. Individuation is something that stems from existence, as was indicated to in the previous proof. Thus, individuation follows quiddity. However, quiddity, in and of itself, is something universal, capable of being predicated for all of its members. Thus, the question arises as to why a particular individual is created following the 
quiddity and not others. There is no definite reason to say why this is so and therefore this theory leads to the contradiction of the law that if there are many possible options that are equal, there is no reason for one of them to be chosen and not the other.

\section{Objection}

If existence is what is essentially created, then the same objection might occur.

Answer

The same objection will not occur. The reason for this is that according to Mulla Sadra existence is essentially individual. Thus, if we say that existence is what is essentially created, then something essentially individual will be what has been primarily created. There will be no need for us to look for the reason as to why this individual has been created to the detriment of the other. It is meaningless to say that something that is essential has a cause from something outside of it. Also, when quiddity follows from existence, something universal is following from something particular. Thus, the abovementioned objection does not occur here as well. This is because a particular is not equally related to all universals. Rather, it is particularly related to the universal that it is an instant of. However, if we start from the universal and move to one individual, the objection occurs because the universal is equally related to all of its individual members.

\section{The Fourth Proof: The Reality of Accidents is Equal to their Reality for their Subjects}

If quiddity is what is essentially created by a cause, then in order for a subject to be predicated with an accident it would be enough for the accident to occur in and of itself and there would be no need for one to take its existence into consideration. However, in order for one to predicate an accident for its subject one must take its existence into consideration. It is not enough for us to take its quiddity into consideration. Thus, quiddity is not what has been essentially created (Mulla Sadra 2006: 186).

\section{Introduction}

In order for us to properly understand this proof it is necessary to first explain something regarding accidents. Beings can be divided into four ba- 
sic categories. Beings can first be divided into beings that are necessary and those that are possible. Following this, possible beings can be divided into beings that are independent and those that are copulative. The beings that are independent can be divided into those that are substances and those that are accidents. Accidents and substances both have independent existences. The difference between them is that the independent existence of the accident is the same as its existence for its substance. This means that although accidents have an independent existence that can be conceived of in the mind independently of something else, when they exist in the world they exist in a locus that does not need them in order to exist. For example, color is an accident that can be conceived independently of anything else. Nevertheless, when color exists in the world it exists in a physical body and it is impossible for it to exist outside of it. The philosophers have stated this matter in the following manner: "The existence of the accident, in and of itself (fi nafsihi), is the same as its existence for its locus (li ghayrihi)". This is in contrast to the copulative existence that does not have an existence, in and of itself. This matter has also been stated in the following manner: When an accident comes into existence in the external world it qualifies something else. For example, when we say that whiteness exists in the external world it implies that something is white. It makes the thing that it exists in white. Thus, its existence performs two functions: It not only removes non-existence from its own quiddity but what is more it removes non-existence from a quality of another being. Meaning, the existence of whiteness not only removes non-existence from the quiddity that is "whiteness" but it also removes non-existence from the quality of "being white" for the locus in which it inheres.

Keeping in mind what was just stated above we can formulate the abovementioned proof in the following manner:

1. If quiddity is what is essentially created by the cause, then it would imply that quiddity is what is essentially real in the outside world.

The proponents of the view that it is quiddity that is essentially created by the cause are of the opinion that existence is an abstract of the mind that is used by the mind to relate the reality of quiddity. Thus, existence is not something real. When a cause performs the function that it does it brings its effect into reality. Thus, if we say that the cause brings quiddity into reality - not existence - it would imply that it is quiddity that is real in the external world not existence. This is like saying that Zayd brought Amr not Bakr into the room. This statement implies that it is Amr that exists in the room.

2. Quiddity is not essentially related to anything else. 
Quiddity is such that it is only what it is. When we take quiddity into consideration, in and of itself, it is only predicable for itself and all other things and qualities that lie outside of the parameters of its boundaries must be negated from it. When we take quiddity, in and of itself, as the subject of a proposition, the only things that can be predicated for it are its essence and its essential parts. Anything outside these parameters must be negated from it.

3. If quiddity is what is essentially created by the cause, then this would imply that the quiddity of an accident is what is created by its cause and it is what is real in the external world.

This premise is the natural conclusion of the first premise.

4. If it is the quiddity of the accident that is real in the external world and quiddity is such that it is not essentially related to anything else, then the quiddity of the accident is not related to anything else in the external world.

This premise is the natural conclusion of the third premise.

5. If the quiddity of the accident is not related to anything in the external world, it will not be related to its locus in the external world.

This premise, in essence, is an instance of the fourth premise.

6. So, if the quiddity of the accident is not related to its locus in the external world, then its independent existence is not the exact same thing as its existence for its locus. Thus, it is not enough for it to exist in order for it to qualify its locus.

In other words, we are faced with a dilemma. Either we can say that it is only quiddity that is created by the cause and say that the quiddity is such that its occurrence in the external world is the same as its occurrence for its locus. If we choose this option, then it would entail that quiddity, in and of itself, can be predicated for something else other than itself. In this case we would have contradicted the philosophical idiom that states that a quiddity taken in and of itself is nothing other than itself. The other option is for us to say that when the cause creates quiddity and brings it into reality it has nothing to do with its locus. In this case we are negating the philosophical axiom that states that the occurrence of quiddity in the external world is the same as its occurrence for its locus. Thus, the idea that quiddity is what has been essentially created by the cause leads to one of two impossible options. Thus, this idea must be negated. 


\section{The Fifth Proof: Quiddity can be Separately Conceived of without Reality}

If quiddity is what is essentially created by the cause, then it would be impossible for one to conceive of a quiddity without conceiving that it was real. However, it is possible to conceive of a quiddity and to be oblivious of the fact that it is real or not. Thus, quiddity is not what has been essentially created by the cause (Mulla Sadra 2006: 186).

1. If we say that it is quiddity that has been essentially created by the cause, then it would imply that quiddity is essentially real.

The reason for this ruling is clear. In the opinion of the adherents of the idea that the cause essentially creates quiddity it is quiddity that therefore is essentially real. Quiddity is what essentially has objective reality and this objectivity is related by the mind in the form of a proposition the predicate of which is existence. Thus, the concept of existence does not essentially have objective reality. Rather, it is simply used to convey the objectivity of quiddity. Since it is quiddity that has objective reality and a cause always brings something - i.e. the effect - into reality, it follows that quiddity is what the cause brings into reality. Conversely, since the cause brings something into reality, if we say that its effect is quiddity it will follow that quiddity is real. In this premise we see the direct relation between the problem of the principality of existence vs. quiddity, and the principality of the creation of existence vs. quiddity. Whatever stance we take in one of the problems will have an effect on the other and vice versa. Nevertheless, this does not imply that there is a viscous circle or argumentation involved since some of the proofs for one of the problems do not rely on the stance we take in the other one. In any case, if we say that quiddity is essentially the effect of the cause, it will lead to the idea that quiddity is what is essentially real in the external world.

2. If quiddity is what is essentially real in the external world and it has not taken its reality from an existee, then being real is a property of quiddity that cannot be separated from it.

The proponents of the principality of existence agree with the idea that quiddity is real. Nevertheless, they say that quiddity has taken its reality from existence. However, the adherents of the principality of quiddity say that reality is essentially a property of quiddity and that the reality of existence is taken from quiddity. If we say that reality is an essential property of quiddity, then it implies that being real is one of the properties of quiddity that cannot separate from it. This is self-evident, since every essential property always 
accompanies the subject for which it is essential. Otherwise, it would not be essential for it.

3. If being real is a property of quiddity that does not separate from it, then when quiddity comes into the mind - and it indeed does come into the mind - then it should be accompanied by this property.

One of the principles of Islamic philosophy is the idea of mental existence (Razi 2008: I/130). This is a cornerstone of Islamic philosophy that we see reflected in many places. One of those places is here. The adherents of mental existence are of the opinion that quiddity enters the mind exactly as it is in the external world. If there is a difference between the mind and reality it is because the mind is not able to fathom existence as it is in the external world. Keeping this in mind, if we say that being real is the essential property of quiddity and it does not separate from it, then when quiddity comes into the mind it must necessarily follow that this property also comes into the mind along with the quiddity.

4. However, we sometimes conceive of quiddity even though we are oblivious of whether the quiddity is real or not.

There is an elaborate discussion in Islamic philosophy regarding the difference between quiddity and existence in the mind. Many of the Sunni theologians are of the opinion that when we predicate existence for any subject then it means exactly the same thing as the subject. The majority of Muslim philosophers, however, are of the opinion that existence has one meaning whereas quiddity has numerous meanings in proportion to the number of propositions in which its existence is predicated. In any case, in order to prove their claim many of the philosophers have presented the following proof: sometimes we conceive of a quiddity and are oblivious of whether it is real or not. If reality and existence were the same thing as quiddity or one of its essential properties, then this would be impossible. Thus, reality is not one of the essential properties of quiddity.

Conclusion: Quiddity is not what has been essentially created by the cause.

\section{Critique}

It seems that one of the premises that this argument rests upon is the idea that quiddity comes into the mind - if not exactly as it is in the external world, then at least as a concept that reflects its nature in the external world. If it does come into the mind in this manner and reality is one of its essential properties, then it would follow that the mind must conceive of this reality 
along with all of its essential properties. However, we are of the opinion that the proof for such an idea rests upon shaky ground. The reason for this is that this proof moves in the following logical manner:

1. There are only two properties of beings in the external world. Those properties are quiddity and existence.

2. Existence does not enter the mind.

3. If quiddity did not enter the mind as it is in reality then it would lead to sophism.

4. However, sophism is wrong. Thus, quiddity enters the mind exactly as it is in the external world.

One of the premises of this proof is the idea that existence does not enter the mind exactly as it is in the external world. Previously, we have discussed the grounds upon which this idea stands and its incorrectness. If existence can come into the mind, there is no need for quiddity to come into the mind as it is in the external world in order for us to save ourselves from falling into sophism. If this is so it is possible for us to say that quiddity leaves one of its essential properties when it comes into the mind, i.e. being real, and it is for this reason that we are able to conceive it without taking into consideration the idea that it is real. What is more, another one of the premises upon which the abovementioned discussion on mental existence rests is the idea that sophism is impossible. However, when we turn to the proofs that have been put forward to substantiate this claim, we see that they only prove that absolute sophism is impossible. This implies that it is impossible to say that we do not have any knowledge of anything at all and that all of our knowledge is wrong. If this is all that this proof shows us, then we cannot conclude from the idea that existence does not come into the mind - assuming that this is true - that quiddity must come into the mind. This is because even if neither of them comes into the mind, it will not lead us to absolute sophistry. This is because there is another form of knowledge that is possible of being true in which there is no room for the concepts of existence and quiddity. That is knowledge by presence. It is possible for us to say that all forms of acquired knowledge are incorrect and that neither quiddity nor existence comes into the mind and still not fall into absolute sophism if we say that knowledge by presence is not incorrect. 


\section{The Sixth Proof: Quiddities Fall under Different Categories}

If quiddity is what is essentially created, then it would mean that all quiddities would fall under the category of relations. However, this is not true. Thus, quiddity is not what is essentially created (Mulla Sadra 2006: 187).

1. If quiddity is what is essentially created, then it would imply that every quiddity would fall under the category of relations.

The idea behind this premise is that being an effect is the relation between the essence of the effect and the essence of the cause. If we say that quiddity is what is essentially related to the cause, then this would imply that it is what essentially has this relation with the cause. Thus, this relation would essentially belong to the quiddity. If we say that it essentially belongs to the quiddity, then it would mean that this relation would be one of the essential parts of the quiddity. If we say that it is one of its essentially parts, then it would imply that it falls under the category of relations. Thus, if we say that it is quiddity that has been essentially created by the cause, then it would imply that all quiddities would fall under the category of relations.

2. However, not all quiddities fall under the category of relations.

This is something that has been proven in its own proper place. By way of example, in the discussions on the soul it has been demonstrated that the human soul is a substance. Even if we only had this example it would be enough to disprove this general rule.

Conclusion: Quiddity has not been essentially created.

\section{Critique}

Although we agree with the major premise of this argument we do not feel that the minor premise is definite. The reason for this is that the idea that quiddity is what is essentially created does not lead to all quiddities falling under the category of relations. The reason for this is that in order for a quiddity to fall under a specific category it is necessary that the category in question be the genus of the quiddity (Tabatabai 2006: 338). However, this does not necessarily entail from the idea that quiddity is what has been essentially created. This is because when we say "essentially" it simply implies that quiddity has not taken this quality from existence. Rather, it stems from quiddity itself. Thus, this term could be taken to be tantamount to a "necessary quality". It is true that the essential parts of a quiddity are necessary for it but the reverse is not true, i.e. it is not true to state that everything that is necessary for a quiddity is one of its 
essential parts. It is equally possible for it to lie extraneous to its essence and nevertheless be necessary for it. Secondly, it is obvious that even if we accepted the necessity between being essentially created and falling under the category of relations, we still have a problem understanding the necessary relationship between the precedent and the antecedent of the minor premise given that not all quiddities have been created in the first place.

\section{Seventh Proof: There is No Gradation in Quiddity}

If quiddity is what has been essentially created by a cause, then it will lead to gradation in quiddity. However, there is no gradation in quiddity. Thus, quiddity has not been essentially created (Mulla Sadra 2006: 187).

1. If quiddity is what is essentially brought into existence, then it will be the effect and the quiddity of its cause will be the cause.

Sometimes we see that one substance is the cause of another. For example, one Intellect is the cause of another Intellect in the vertical hierarchy of Intellects. If we say that quiddity is what is essentially brought into reality by its cause, this would mean that the effect of the higher Intellect is the quiddity of the lower Intellect. It would also imply that the cause of the lower Intellect is the quiddity of the higher Intellect. The emphasis of this premise is that, if we say that quiddity is the effect of the cause, then we also have to say that it is the quiddity of the cause that is essentially the cause of the effect. There is a subtle reason why this is so. This is because the thing that has been essentially created by the cause is also essentially real. This is the connection between the problem of what is principle in creation and what is principle in reality. If we say that it is quiddity that is essentially created by the cause, then it would necessitate that it is also what is essentially real, since these two problems are necessary corollaries of one another. When we say that it is the quiddity of the effect that is essentially real then we have no choice but to say that it is the quiddity of the cause that is also real. This is because we cannot adhere to the idea that in some beings quiddity is real while in other beings existence is real. In other words, if something is essentially real it will be so all the time. It is meaningless to say that something is essential for something else and at the same time to say that it forgoes that quality that is essential for it sometimes. This is a blatant contradiction.

2. There is a type of gradation between cause and effect.

The reality of the cause precedes the reality of the effect and the reality of the effect follows the reality of the cause. What is more, the reality of the cause is stronger than the reality of the effect. 
3. If we say that cause and effect are two quiddities, then it means that there is gradation between quiddities.

This premise is the natural conclusion of the previously mentioned premises.

4. However, there is no such thing as gradation between two quiddities.

Out of all of the premises of this argument this seems to be the most vital. Gradation is a subject with a long history in Muslim philosophy and one that has seen some major upheavals throughout time. Nevertheless, prior to Sheikh Ishraq the majority of Peripatetic philosophers were strongly of the opinion that there is no such thing as gradation in quiddity. Since entering this discussion would lead us too far astray from the original discussion we will simply ask the reader to take this for granted at this point.

Conclusion: Quiddity is not what is essentially created by the cause.

\section{Conclusion}

The problem of what is essentially created by a cause is one that is necessarily related to the problem of what is essentially real. Any conclusion that we reach in one of these discussions will necessarily have an effect on the other. On the other hand, Mulla Sadra has presented sufficient proofs to corroborate the idea that existence is essentially real. This should be enough to show that it is what is essentially created by a cause. Nevertheless, Mulla Sadra has also presented many independent proofs to prove this idea that do not rely on the principality of existence. Nonetheless, some of these proofs share some premises with the proofs for the principality of existence. As we have seen in this article, some of these are problematic as they contradict other accepted philosophical principles and others are sound. All in all we can say that the principality of the creation of existence is an unassailable philosophical principle.

Received: June $29^{\text {th }}, 2016$.

Accepted: July $27^{\text {th }}, 2016$.

\section{Bibliography}

Fazlurahman (1975), The Philosophy of Mulla Sadra, New York, State University of New York Press.

Fayyadhi, Ghulam Ridha (2007), Daramadi bar Marefatshenasiye Eslami, Qom, Mu'assasa Imam Khomeini. 
Fayyadhi, Ghulam Ridha (2008), Hasti wa Chisti dar Maktabe Sadrai, Qom, Pazhuheshgahe Howzah wa Daneshgah.

Misbah, Muhammad Taqi (1999), Philosophical Instructions, New York, Global Publications.

Mulla Sadra, Muhammad ibn Ibrahim (1963), al-Asfar, Qom, Dar al-Ma’arif al-Islamiyyah.

Mulla Sadra, Muhammad ibn Ibrahim (2006), Majmu' ar-Rasail al-Falsafiyyah, Tehran, Hikmat Publications, $3^{\text {rd }}$ Edition.

Razi, Muhammad ibn Umar (2008), al-Mabahith al-Mashriqiyyah, Qom, Bidar, $2^{\text {nd }}$ Edition.

Tabatabai, Muhammad Husain (2006), Nihayat al-Hikmah, Qom, Mu'assasa Imam Khomeini.

Tabatabai, Muhammad Husain (2010), Bidayat al-Hikmah, Qom, Jami’a al-Mudarrisin Publications.

Ubudiyyat, Abd al-Rasul (2013), Nezame Hekmate Sadrai, Qom, Mu'assasa Imam Khomeini, $6^{\text {th }}$ Edition. 